\title{
Finite Element Method withPiecewise Linear Function for Solving NanoscaleInAs/GaAsQuantum RingStructures
}

\author{
Eman Ali Hussain ${ }^{1}$, Jamil A. Al-Hawasy ${ }^{2}$ and Lamyaa H. Ali ${ }^{3}$ \\ ${ }^{1}$ Department of Mathematics, college of Science, University of Al-Mustansiriyah, Iraq \\ E-mail: "dreman@uomustansiriyah.edu.iq,dr_emansultan@yahoo.com" \\ ${ }^{2}$ Department of Mathematics, college of Science, University of Al-Mustansiriyah, Iraq \\ E-mail:jhawassya@uomustansiriyah.edu.iq, hawasy20@yahoo.com \\ ${ }^{3}$ Department of Mathematics, college of Science, University of Al-Mustansiriyah, Iraq \\ E-mail:"lamya_h2@uomustansiriyah.edu.iq, lamya_h2@yahoo.com"
}

\begin{abstract}
In this paper concerned with the solution of the nanoscale structures consisting of the InAs/GaAswith an effective mass envelope function theory, the electronic states of the InAs/GaAs quantum ring are studied.In calculations, the effects due to the different effective masses of electrons in and out the rings are included. The energy levels of the electron are calculated in the different shapes of rings, i.e., that the inner radius of rings sensitively change the electronic states. The energy levels of the electron are not sensitively dependent on the outer radius for large rings. The structures of InAs / GaAs quantum rings are studied by the one electronic band Hamiltonian effective mass approximation, the energy-and position-dependent on electron effective mass approximation, and the spin-dependent on the Ben Daniel-Duke boundary conditions. In the description of the Hamiltonian matrix elements, the Finite elements method with different base piecewise linear function is adopted. The non-linear energy confinement problem is solved approximately by using the Finite elements method with piecewise linear function, to calculate the energy of the one electron states for the InAs/GaAs quantum ring. The results of numerical example are compared for accuracy and efficiency with the finite element method of linear triangular element. This comparison shows that good results of numerical example.
\end{abstract}

[DOI: $10.22401 /$ ANJS.00.2.17]

Keywords: Nanoscale, Finite elements method, Ben Daniel-Duke boundary conditions, InAs/GaAs quantum rings.

\section{Introduction}

The modeling of the electron states in semiconductor nanostructures remains a difficult computational task. The one electron states are helpful for studying the electron correlations and, the effects of magnetic fields in quantum rings and useful for designing and fabricating the double colors detector by intra band and inter band translations.

In 1990, Paaschet al., [3] used envelope equation and wave function matching for narrow-gap semiconductors. In 1995, Mathine et al., [4] applied computational Fourier series solution of the BenDaniel-Duke Hamiltonian for arbitrary shaped quantum wells. In 2001,Yiming Li et al., [1] usedComputer simulation of electron energy levels for different shape InAs/GaAs semiconductor quantum dots. In 2002, Yiming Li et al, [2] used Electron energy state spin-splitting in 3D cylindricalsemiconductor quantum dots. In 2003,Melnik et al, [5]applied finite element analysis of Nanowire superlattice structures.Whereas2005, Yiming Li,[6] using an iterative method for single and vertically stacked semiconductor quantum dots simulation. In 2016,Deyasi et al., [7]applied numerically computed in presence of electric field using propagation matrix method.In 2017, Emanet. al., [8] using finite element method withlinear triangular element for solving finite nanowire superlattice quantum dot structures GaAs/AlGaAs. In 2017, Eman et al., [9] using finite element method withlinear rectangular element for solving finite nanowire superlattice quantum dot structures GaAs/AlGaAs .

In this paper we propose a Finite element method with different base piecewise linear function for solving the nanoscale structures 
consisting of the InAs/GaAs quantum ring, and the spin-dependent on the Ben DanielDuke boundary conditions.

\section{Modeling Energy Stat With Spin- Dependent Boundary Conditions [5],[6]}

We consider the problem to compute relevant energy states and corresponding wave functions of a three dimensional semiconductor quantum ring. Consider the oneelectronsis confined in system of the three-dimensional quantum ring structures and apply an effective one electronic band Hamiltonian, is given by:

$\widehat{H}=\widehat{H}_{0}+\widehat{V}_{s o}(r)$

where $\widehat{H}$ is the Hamiltonian of the system without spin-orbit interaction, $\widehat{V}_{s o}(r)$ is the spin-orbit interaction for the conduction band electrons, andthe expression for $\widehat{H}_{0}$ is as follows:

$\widehat{H}_{0}=-\frac{\hbar^{2}}{2} \nabla_{r}\left(\frac{1}{m(E, r)}\right) \nabla_{r}+V(r)$

where $\nabla_{r}$ is the spatial gradient, $m(E, r)$ is the energy dependent electron effective mass, and $V(r)$ is the confinement potential.

$$
\begin{aligned}
\frac{1}{m(E, r)}=\frac{P^{2}}{\hbar^{2}} & {\left[\frac{2}{E+E_{g}(r)-V(r)}\right.} \\
& \left.+\frac{1}{E+E_{g}(r)+\Delta(r)-V(r)}\right]
\end{aligned}
$$

where $E_{g}(r)$ and $\Delta(r)$ stand for the position dependent band gap and the spin-orbit splitting in the valence band, respectively and, $P$ is the momentum matrix element.

The spin-orbit interaction for the conduction band electrons $\widehat{V}_{s o}(r)$ is given by: $\widehat{V}_{s o}(r)=i \nabla \beta(E, r) \cdot[\hat{\sigma} \times \nabla]$

where $\beta(E, r)$ is the spin-orbit coupling parameter and $\hat{\sigma}=\left\{\sigma_{x}, \sigma_{y}, \sigma_{z}\right\}$ is the vector of the Pauli matrices. The energy and position dependent $\beta(E, r)$ has the form:

$$
\begin{aligned}
\beta(E, r)=\frac{P^{2}}{2}[ & \frac{1}{E+E_{g}(r)-V(r)} \\
& \left.-\frac{1}{E+E_{g}(r)+\Delta(r)-V(r)}\right]
\end{aligned}
$$

For those quantum ring systems that have sharp discontinuity on the conduction band interfaces between the quantum ring ("InAs" material 1) and semiconductor matrix
("GaAs" material 2), the hard-wall confinement potential is:

$V(r)=\left\{\begin{array}{c}0, r \in \text { material } 1 \\ V_{0}, r \in \text { material } 2\end{array}\right.$

where $V_{0}$ is the structure band offset. Combining the Hamiltonian in equations (1), (2), and (4), the spin dependent Ben DanielDuke boundary conditions for the electron wave function $\Psi(r)$ is written as follows:

$$
\begin{gathered}
\Psi_{\text {material } 1}\left(r_{S}\right)=\Psi_{\text {material } 2}\left(r_{S}\right) \\
\left\{\frac{\hbar^{2}}{2 m(E, r)} \nabla-i \nabla \beta(E, r)[\widehat{\sigma} \times \nabla]\right\}_{n} \Psi\left(r_{S}\right)=\mathrm{C}_{0}
\end{gathered}
$$

where $V_{0}$ is the some constant, $r_{s}$ denotes the position of the system interface.

Note (1): We note that the expressions of electron effective mass in equation (3), spinorbit coupling parameter in equation (5), and the equations of Ben Daniel-Duke boundary condition in equation (7) are all energy and position dependent relationships in this study.

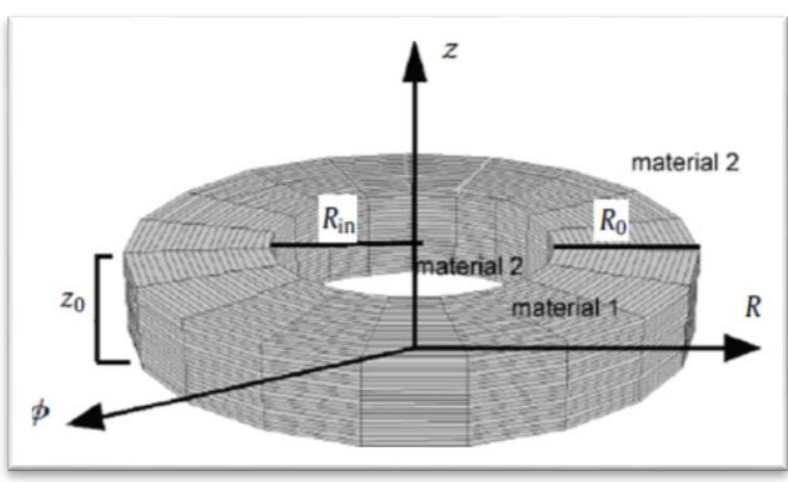

Fig.(1) A three-dimensional plot of the disk shaped semiconductor quantum ring.

We now consider the quantum ring as shown in Fig.(1) with the inner radius $R_{i n}$, radius $\mathrm{R}_{0}$ and the thickness $\mathrm{Z}_{0}$ in the cylindrical coordinate $(R, \phi, Z)$. The origin of the system is at the center of the structure and the $\mathrm{Z}$ axis is chosen along the rotation axis. Since the system is cylindrically symmetric, the wave function can be represented as:

$\Psi(r)=\phi(R, Z) \exp (i l \phi)$

wherel isa constant represents the electron orbital quantum number and the original model remains a two-dimensional problem in $(R, Z)$ coordinate. From equations (1)-(6) and equation (8), we obtain the following equation:

$-\frac{\hbar^{2}}{2 m_{1}(E)}\left(\frac{\partial^{2}}{\partial R^{2}}+\frac{\partial}{R \partial R}+\frac{\partial^{2}}{\partial Z^{2}}-\frac{l^{2}}{R^{2}}\right) \phi_{1}(R, Z)=$ $E \phi_{1}(R, Z), \forall(R, Z) \in$ material 1 
and

$$
\begin{aligned}
& -\frac{\hbar^{2}}{2 m_{2}(E)}\left(\frac{\partial^{2}}{\partial R^{2}}+\frac{\partial}{R \partial R}+\frac{\partial^{2}}{\partial Z^{2}}-\frac{l^{2}}{R^{2}}\right) \phi_{2}(R, Z)+ \\
& V_{0} \phi_{2}(R, Z)=E \phi_{2}(R, Z), \forall(R, Z) \in \\
& \text { material } 2
\end{aligned}
$$

For the same reasons that the problem is symmetry along the $\mathrm{Z}$ axis, the spindependent boundary conditions in equation (7) are given by:

$$
\begin{aligned}
& \phi_{1}(R, Z)=\phi_{2}(R, Z), Z=f(R) \\
& \text { and } \\
& \frac{1}{m_{1}(E)}\left\{\frac{\partial \phi_{1}(R, Z)}{\partial R}+\frac{d f(R)}{d R} \frac{\partial \phi_{1}(R, Z)}{\partial R}\right\}_{Z=f(R)}- \\
& \frac{1}{m_{2}(E)}\left\{\frac{\partial \phi_{2}(R, Z)}{\partial R}+\frac{d f(R)}{d R} \frac{\partial \phi_{2}(R, Z)}{\partial R}\right\}_{Z=f(R)}+ \\
& \frac{2 \sigma\left(\beta_{1}-\beta_{2}\right)}{\hbar^{2}} \frac{l}{R_{0}} \phi_{1}\left(R_{0}, Z\right)=0
\end{aligned}
$$

\section{Finite Element Method}

Dependence of the electron effective mass and spin-orbit coupling parameter on each energy state results in a nonlinear equations (9) and (10). The nonlinear equations complicate the process of analytical solution in the explored quantum ring. Therefore, the numerical approach to the solution of the nonlinear equations is advanced in the calculation of the electronic structure of InAs / GaAs quantum rings. The finite element method is applied to solve the above problem for the nanoscale InAs / GaAs quantum rings. Energy states and spinsplitting are numerically calculated without any fitting parameters. Starting from a given initial energy, the finite element method globally calculates all bounded energies for the corresponding nonlinear algebraic eigenvalue problem. A computational procedure of the finite element method is shown below:

$$
-\frac{\hbar^{2}}{2 m_{i}(E)}\left(\frac{\partial^{2}}{\partial R^{2}}+\frac{\partial}{R \partial R}+\frac{\partial^{2}}{\partial Z^{2}}-\frac{l^{2}}{R^{2}}\right) \phi_{i}(R, Z)+
$$$$
V \phi_{i}(R, Z)=E \phi_{i}(R, Z), \forall(R, Z) \in
$$

material $i$

where $i=1,2$ since the cylindrical is symmetry, $\quad V=V(R, Z), \quad$ and $\phi_{i}=$ $u(R, Z) e^{i l \phi_{i}}$, where $l$ is an integer. Equation (12) becomes

$\frac{-\hbar^{2}}{2 m_{i}(E)}\left[\frac{1}{R} \frac{\partial u}{\partial R}+\frac{\partial}{\partial R}\left(\frac{\partial u}{\partial R}\right)-\frac{l^{2}}{R^{2}} u+\frac{\partial}{\partial Z}\left(\frac{\partial u}{\partial Z}\right)\right]+$

$V u=E u$

The electron energy spectra for InAs / GaAs quantum rings the semiconductor band structure governing physical process in a ring nanoscale are described in terms of cylindrical coordinates. When the geometry, loading, and boundary conditions are independent of the circumferential direction $(\phi$-coordinate), the electron energy spectra for InAs/GaAs quantum rings of the semiconductor band structure equation become two-dimensional in terms of $R$ and $Z$.

\subsection{Weak Form}

Assume that $\Omega$ is a typical element the piecewise linear function of the finite element mesh, and we develop the finite element model of equation (13) over $\Omega$. Various twodimensional elements will be discussed in the sequel.

There are three steps in the development of the weak form of equation (13) over the typical element $\Omega$.

The first step is formulated equation (13) by the following weak form.

$$
\begin{aligned}
0= & \int_{\Omega}\left[\frac { - \hbar ^ { 2 } } { 2 } \left[w \frac{1}{m_{i}(E)} \frac{\partial u}{\partial R}+w R \frac{\partial F_{1}}{\partial R}-\right.\right. \\
& \left.\frac{l^{2}}{m_{i}(E) R} w u+w R \frac{\partial F_{2}}{\partial Z}\right]+V w R u- \\
& E w R u] d R d Z
\end{aligned}
$$

where:

$$
F_{1}=\left(\frac{1}{m_{i}(E)} \frac{\partial u}{\partial R}\right) \operatorname{and} F_{2}=\left(\frac{1}{m_{i}(E)} \frac{\partial u}{\partial Z}\right)
$$

In the second step, we note the identities:

$$
\begin{aligned}
& -w R \frac{\partial F_{1}}{\partial R}=R \frac{\partial w}{\partial R} F_{1}-R \frac{\partial}{\partial R}\left(w F_{1}\right) \\
& -w R \frac{\partial F_{1}}{\partial Z}=R \frac{\partial w}{\partial Z} F_{2}-R \frac{\partial}{\partial Z}\left(w F_{2}\right)
\end{aligned}
$$

Next, using equation (15a) and equation (15b) in the equation (14) then applying the divergence theorem, we obtain:

$$
\begin{aligned}
0= & \int_{\Omega}\left[\frac{-\hbar^{2}}{2 m_{i}} w \frac{\partial u}{\partial R}+\frac{\hbar^{2}}{2} R \frac{\partial w}{\partial R}\left(\frac{1}{m_{i}} \frac{\partial u}{\partial R}\right)+\right. \\
& \frac{\hbar^{2} L^{2}}{2 m_{i} R} w u+\frac{\hbar^{2}}{2} R \frac{\partial w}{\partial z}\left(\frac{1}{m_{i}} \frac{\partial u}{\partial Z}\right)+w R V u- \\
& w R E u] d R d Z-\oint_{\Gamma} w\left[n_{1}\left(\frac{1}{m_{i}} \frac{\partial u}{\partial z}\right)+\right. \\
& \left.n_{2}\left(\frac{R}{m_{i}} \frac{\partial u}{\partial R}\right)\right] d s
\end{aligned}
$$

where $\vec{n}=\left(n_{1}, n_{2}\right)$ is an outer unite vector normal on $\Gamma$ and $d s$ is the length of an infinitesimal line element along the boundary.

From an inspection of the boundary integral in (16), we note that the specification of $u$ constitutes the essential boundary condition, and hence $u$ is the primary variable. The specification of the coefficient 
of the weight function in the boundary expression, i.e., we can let:

$d_{n} \equiv n_{1}\left(\frac{1}{m_{i}} \frac{\partial u}{\partial Z}\right)+n_{2}\left(\frac{R}{m_{i}} \frac{\partial u}{\partial R}\right)$

The third and last step of the formulation is to substitute the definition (17) in (16) and write the weak form of (13) as:

$$
\begin{aligned}
0= & \int_{\Omega}\left[\frac{-\hbar^{2}}{2 m_{i}} w \frac{\partial u}{\partial R}+\frac{\hbar^{2}}{2} R \frac{\partial w}{\partial R}\left(\frac{1}{m_{i}} \frac{\partial u}{\partial R}\right)+\right. \\
& \frac{\hbar^{2} L^{2}}{2 m_{i} R} w u+\frac{\hbar^{2}}{2} r \frac{\partial w}{\partial Z}\left(\frac{1}{m_{i}} \frac{\partial u}{\partial Z}\right)+w r V u- \\
& w r E u] d R d Z-\oint_{\Gamma} w d_{n} d s
\end{aligned}
$$

Now, $u(R, Z)$ is approximated over a typical finite element $\Omega$ by the expression: $u(R, Z) \approx u_{h}^{e}(R, Z)=\sum_{j=1}^{n} u_{j}^{e} \psi_{j}^{e}(R, Z)$

where $u_{j}^{e}(R, Z)$ is the value of $u_{h}^{e}(R, Z)$ at the $j^{\text {th }}$ node $\left(R_{j}, Z_{j}\right)$ of the element .

Substituting the finite element approximation (19) for $u$ into the weak form (18), we get:

$$
\begin{aligned}
0= & \int_{\Omega}\left[\frac{-\hbar^{2}}{2 m_{i}} w \sum_{j=1}^{n} u_{j}^{e} \frac{\partial \psi_{j}^{e}}{\partial R}+\right. \\
& \frac{\hbar^{2}}{2} R \frac{\partial w}{\partial R}\left(\frac{1}{m_{i}} \sum_{j=1}^{n} u_{j}^{e} \frac{\partial \psi_{j}^{e}}{\partial R}\right)+ \\
& \frac{\hbar^{2} l^{2}}{2 m_{i} R} w \sum_{j=1}^{n} u_{j}^{e} \psi_{j}^{e}+ \\
& \frac{\hbar^{2}}{2} R \frac{\partial w}{\partial Z}\left(\frac{1}{m_{i}} \sum_{j=1}^{n} u_{j}^{e} \frac{\partial \psi_{j}^{e}}{\partial Z}\right)+ \\
& w R V \sum_{j=1}^{n} u_{j}^{e} \psi_{j}^{e}- \\
& \left.w R E \sum_{j=1}^{n} u_{j}^{e} \psi_{j}^{e}\right] d R d Z-\oint_{\Gamma} w d_{n} d s
\end{aligned}
$$

This equation must hold for every admissible choice of weight function $w$. Since we need $n$ independent algebraic equations to solve for the $n$ unknowns, $u_{1}^{e}, u_{2}^{e}, \ldots, u_{n}^{e}$, we choose $n$ linearly independent functions for $w: w=\psi_{1}^{e}, \psi_{2}^{e}, \ldots, \psi_{n}^{e}$. For each choice of $w$ we obtain an algebraic relation among $\left(u_{1}^{e}, u_{2}^{e}, \ldots, u_{n}^{e}\right)$. We label the algebraic equation resulting from substitution of $w=\psi_{i}^{e}$ into (20):

$$
\begin{aligned}
0= & \sum_{j=1}^{n}\left\{\int _ { \Omega } \left[\frac{-\hbar^{2}}{2 m_{i}} \psi_{i}^{e} \frac{\partial \psi_{j}^{e}}{\partial R}+\right.\right. \\
& \frac{\hbar^{2}}{2} R \frac{\partial \psi_{i}^{e}}{\partial R}\left(\frac{1}{m_{i}} \frac{\partial \psi_{j}^{e}}{\partial R}\right)+\frac{\hbar^{2} L^{2}}{2 m_{i} R} \psi_{i}^{e} \psi_{j}^{e}+ \\
& \frac{\hbar^{2}}{2} R \frac{\partial \psi_{i}^{e}}{\partial z}\left(\frac{1}{m_{i}} \frac{\partial \psi_{j}^{e}}{\partial Z}\right)+R V \psi_{i}^{e} \psi_{j}^{e}- \\
& \left.\left.R E \psi_{i}^{e} \psi_{j}^{e}\right] d R d Z\right\} u_{j}^{e}- \\
& \oint_{\Gamma} w d_{n} d s ; i, j=1,2, \ldots, n \ldots(21)
\end{aligned}
$$

\subsection{Finite Element Method for the Model With Piecewise Linear Functions}

In this section, the solution $u$ of equation (18) is approximated by a FEM for the model with piecewise linear function.

We now define $V_{h}$ as follows:

$V_{h}=\{v: v$ is cotinuous on $\Omega, v=0$ on $\Gamma\}$.

The space $V_{h}$ consists of all continuous function that are linear on each triangle $K$ and vanish on $\Gamma$. We notice that $V_{h} \subset V$. As parameters to describe a function $v \in V_{h}$ we choose the values $v\left(N_{i}\right)$ of $v$ at the nodes $N_{i}, i=1, \ldots, N$, of $T_{h}$ but exclude the nodes on the boundary since $v=0$ on $\Gamma$.

The corresponding basis function $\varphi_{j} \in V_{h}, j=1, \ldots, N$, are then defined by (see Fig.(2)):

$\varphi_{j}\left(N_{j}\right)=\delta_{i j} \equiv\left\{\begin{array}{l}1 \text { if } i=j \\ 0 \text { if } i \neq j\end{array} i, j=1, \ldots, M\right.$.

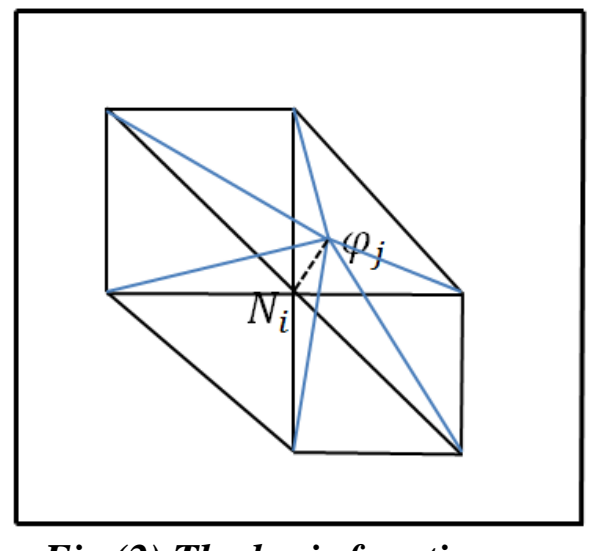

Fig.(2) The basis function $\varphi_{j}$.

The finite element method was applied to project the variational form of the problem onto a finite dimensional space. As a result, the problem was reduced to the following generalized eigenvalue problem:

\section{$A u=\lambda B$}

where $A$ and $B$ are $N \times N$ matrices, $u$ the vector of unknowns of dimensionality $N$, and $N$ the number of nodes in which the solution to the problem (13) is being sought. Computational domains of interest are symmetric with respect to the z-axis, where we imposed Neumann's boundary conditions if $L=0$.The solution to equation (22) was found in MATLAB.

We employed the finite element method so as to calculate electron eigenvalues and eigenstates for a series of finite NWSL structures with a cylindrical cross-section. In 
this case the matrices in equation (22) hos the following form

$$
\begin{aligned}
A_{i j}= & \int_{\Omega}\left[\frac{-\hbar^{2}}{2 m} \psi_{i}^{e} \frac{\partial \psi_{j}^{e}}{\partial r}+\frac{\hbar^{2}}{2} r \frac{\partial \psi_{i}^{e}}{\partial r}\left(\frac{1}{m} \frac{\partial \psi_{j}^{e}}{\partial r}\right)+\right. \\
& \left.\frac{\hbar^{2} L^{2}}{2 m r} \psi_{i}^{e} \psi_{j}^{e}+\frac{\hbar^{2}}{2} r \frac{\partial \psi_{i}^{e}}{\partial z}\left(\frac{1}{m} \frac{\partial \psi_{j}^{e}}{\partial z}\right)\right] d r d z \\
B_{i j}= & \int_{\Omega}[V-E] r \psi_{i}^{e} \psi_{j}^{e} d r d z
\end{aligned}
$$

As a first choice the uniform mesh are used by the piecewise linear function three element, as shown in Fig.( 3) to represent the domain, to determine the element coefficient matrices $A$ and $B$ of Eq. (22).

To evaluation of the integral in Eq. (23) and Eq. (24), it is possible to obtain the closed form for the $A$ and $B$ matrices by carrying out the integrals in Eq. (23) and Eq. (24) exactly respectively. We consider element 1 as the typical element. Hence, the element coefficient $A$ and $B$ matrices are:

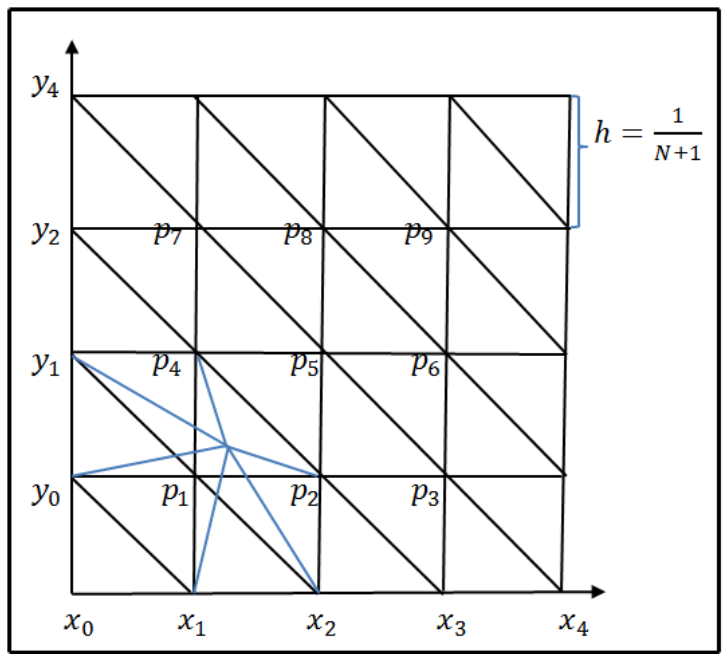

Fig.(3) The uniform mesh piecewise linear function three element.

\section{Notes (1):}

In the following example, the representative set of parameters were used for the calculations as:

For InAs, the energy gap $E_{g}=$ $0.42 \mathrm{eV}, \Delta_{1}=0.38 \mathrm{eV}, m_{1}(0)=0.024 \mathrm{~m}_{0}$. For GaAs, the energy gap $E_{g}=1.52 \mathrm{eV}, \Delta_{1}=$ $0.34 \mathrm{eV}, \mathrm{m}_{1}(0)=0.067 \mathrm{~m}_{0}$. The band offset parameter is taken as $V_{0}=0.55 \mathrm{eV}$.

\section{Example (1):}

Consider the NanoscalInAs/GaAs, quantum ring model, with using the above date to solve InAs/GaAs, nanostructures quantum ring.
In this example the FEM with piecewise linear function we used to solve equation (13).

Equation (13) is solved using MATLAB program at $n=1296$. The results of numerical example are gives a good accuracy and efficiency of this method compare the results with FEM of linear triangular element and the maximum absolute error is less than $10^{-2} \mathrm{eV}$ (shown in Fig.(4)).

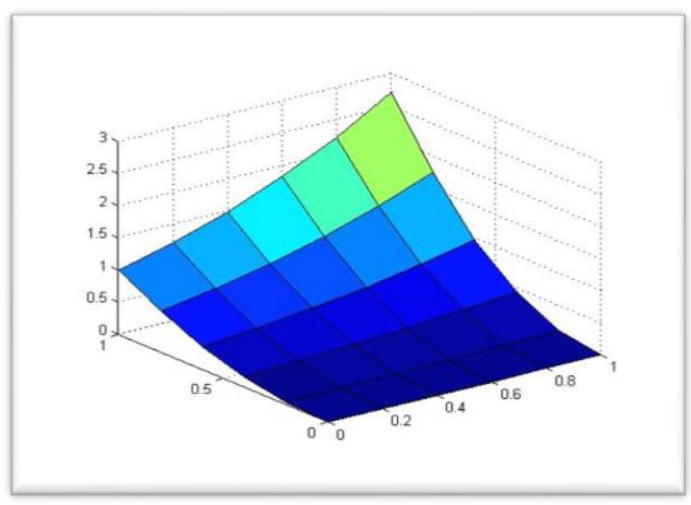

(a)

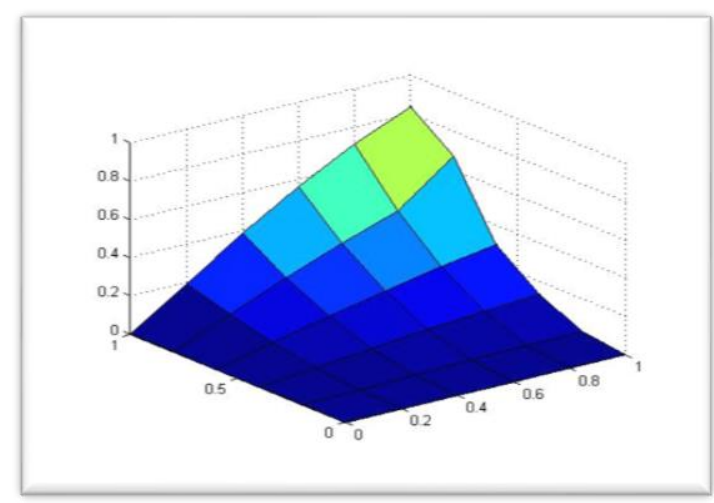

(b)

Fig.(4) The calculators of equation (13), (a) calculated with the FEM piecewise linear function, (b) calculated with the FEM of linear triangular element.

\section{Conclusion}

The finite element method with using different base piecewise linear function are used for solving the nanoscale structures consisting of the InAs/GaAs quantum ring, and the spin-dependent on the Ben DanielDuke boundary conditions. The results of numerical example are gives a perfect accuracy and efficiency of this approach. The results of numerical example are gives a good accuracy and efficiency of this method compare the results with FEM of linear triangular element. 


\section{References}

[1] Yiming Li, O. Voskoboynikov, C.P. Lee, S.M. Sze"Computer simulation of electron energy levels for different shape InAs/GaAs semiconductor quantum dots' Computer Physics Communications, Vol. 141, (2001), pp. 66-72.

[2] Yiming Li, O. Voskoboynikov, C.P. Lee, S.M. SzeO. Tretyak" Electron energy state spin-splitting in 3D cylindrical semiconductor quantum dots' $T$ The European Physical Journal B, Vol. 28, No. 8 (2002), pp. 475-481.

[3] G. Paasch, P. H. Nguyen and G. Gobsch"'Envelope Equation and Wave Function Matching for Narrow-Gap Semiconductors''Physica Status Solidi (b), Vol. 162, No. 1 (1990), pp.155-163.

[4] D. L. Mathine, S. K. Myjak and G. N. Maracas "A Computational Fourier SeriesSolutin of the BenDaniel-Duke Hamiltonian for Arbitrary Shaped Quantum Wells" IEEE Journal of Quantum Electronics, Vol. 31, (1995), pp. 1216-1222.

[5] M. Willatzence, R. Melnik, C. Galeriu and L. C. Lew Yan Voon"Finite Element Analysis of Nanowire Superlattice Structures' Springer, Vol. 31, (2003), pp. 755-763.

[6] Yiming Li, “An Iterative Method for Single and Vertically Stacked Semiconductor Quantum Dots Simulation' 'Mathematical and Computer Modelling, Vol. 42, (2005), pp. 711-718.

[7] D. Sarkara, A. Deyasib" Field Induced Tuning of DOS and Eigenstates in Double Quantum Well Structure having Gaussian Geometry" American Scientific Publishers, Vol. 5,No. 1,(2016), pp. 138-143.

[8] Eman A. Hussain, Jamil A. A. AlHawasy, Lamyaa H. Ali, "Finite Element Method Linear Triangular Element for Solving Finite Nanowire Superlattice Quantum Dot Structures GaAs/AlGaAs" Journal of Computer and Mathematical Sciences, Vol. 8(3), (2017), PP. 96-105.
[9] Eman A. Hussain, Jamil A. A. AlHawasy, Lamyaa H. Ali, "Finite Element Method Linear Rectangular Element for Solving Finite Nanowire Superlattice Quantum Dot Structures GaAs/AlGaAs" The International Journal of Engineering and Science (IJES), Vol. 6(4), (2017), PP. 01-07. 